\title{
Quantification of D-mannose in plasma: development and validation of a reliable and accurate HPLC-MS-MS method.
}

Beatrice Campi ${ }^{1}$, Simone Codini ${ }^{2}$, Niccolò Bisoli ${ }^{3}$, Simona Baldi $^{2}$, Riccardo Zucchi ${ }^{3}$, Ele Ferrannini ${ }^{1}$, Alessandro Saba ${ }^{3,4}$

1. C.N.R. Institute of Clinical Physiology, Pisa, Italy

2. Department of Clinical and Experimental Medicine, University of Pisa, Pisa, Italy.

3. Department of Surgical, Medical and Molecular Pathology and Critical Care Medicine, University of Pisa, Pisa, Italy

4. Laboratory of Clinical Pathology, St. Chiara University Hospital, Pisa, Italy.

Corresponding author: Alessandro Saba

alessandro.saba@med.unipi.it

St. Chiara University Hospital

Via Roma 67, 56126 Pisa, Italy

ORCID: 0000-0003-0611-5549 


\begin{abstract}
The present paper describes the development and the validation process - in compliance with the EMA guidelines - of a method based on tandem mass spectrometry coupled to liquid chromatography for the accurate quantification of mannose in human plasma samples. The quick sample preparation procedure, simplified by the absence of any derivatization step, makes the assay suitable for routine use in a clinical chemistry laboratory. The method validation yielded satisfactory selectivity, with a good separation of mannose from its epimers (glucose and galactose), linearity over the whole concentration range of interest $(0.31-40 \mu \mathrm{g} / \mathrm{mL})$, reproducibility with RSD $<10 \%$, and accuracy in the range $96-104 \%$. Instrumental LLOD $(0.31 \mu \mathrm{g} / \mathrm{mL})$ and LLOQ $(1.25$ $\mu \mathrm{g} / \mathrm{mL}$ ) were good enough to detect endogenous plasma mannose levels and in agreement with recent data from the literature. Sensitivity was affected by a 5-fold dilution factor, which, if necessary, can be reduced. The method robustness was proven in more than 600 injections, most of them being of plasma samples, used also to assess the reference ranges in healthy subjects $(9.93 \pm$ $3.37 \mu \mathrm{g} / \mathrm{mL})$ and type 2 diabetic patients $(23.47 \pm 6.19 \mu \mathrm{g} / \mathrm{mL})$.
\end{abstract}

\title{
Keywords
}

D-mannose, tandem mass spectrometry, HPLC, validation 


\section{Introduction}

Mannose, a sugar monomer of the aldohexose series, is important in mammalian metabolism, especially in humans, where it is involved in the glycosylation of several proteins and plays a causal role in some diseases. In fact, several congenital disorders of glycosylation are associated with mutations in enzymes involved in mannose metabolism [1]. In particular, biochemical and genetic data have shown that genes involved in the O-mannose glycosylation pathway may cause forms of congenital muscular dystrophy in humans [2]. Moreover, mannose has been implicated in cancer metabolism and is considered to be a reliable biomarker for esophageal adenocarcinoma (EAC) $[3,4]$. Moreover, serum concentrations of D-mannose have been reported to be higher in metastatic breast cancer patients than in early-stage breast cancer [5]. Use of this metabolite as a marker for the early detection of both breast and esophageal cancer may improve the clinical outcome of these diseases $[4,5]$. Recent studies have also shown that elevated plasma mannose levels are related with future risk of several chronic diseases, including type 2 diabetes (T2D), cardiovascular disease (CVD), and renal impairment (albuminuria) [6]. Thus, mannose is posited as a new biomarker for the risk of several conditions and their complications, and may also open novel and early therapy.

In the recent literature, methods for the assessment of D-mannose in biological fluids have included enzymatic [7,8,9], high-performance liquid chromatography (HPLC) [10,11], gas-liquid chromatography [12], and capillary electrophoretic techniques [13]. Recently, Miwa and Taguchi [11] reported a valuable HPLC method for the analysis of D-mannose in plasma, which was based on sugar derivatization before detection. Derivatization is a common practice in the field, utilized for gas chromatographic separation or for detection; however, derivatization lengthens sample preparation time and cost considerably, i.e., not the best choice for routine analysis.

We developed and validated an accurate D-mannose assay by tandem mass spectrometry coupled to liquid chromatography (HPLC-MS-MS), capable of discriminating mannose from Dglucose, a C2 epimer of D-mannose, which in humans circulates at a concentration at least 100-fold higher [4]. Compared to traditional techniques, tandem mass spectrometry has enhanced 
selectivity, limited cost of analysis, and allows the determination of compound of interest in biological fluids. Unfortunately, it does not discriminate isobaric compounds such as epimers, which need to be separated by ad hoc liquid chromatography prior the MS detection. In the present study, a HILIC column specific for saccharides analysis was used, which provided a clear separation of D-mannose from its potential interferent compounds, D-glucose and D-galactose.

\section{Materials and method}

\subsection{Reagents and materials}

D-mannose (M) and ${ }^{13} \mathrm{C}_{6}$-D-mannose $\left({ }^{13} \mathrm{C}_{6}-\mathrm{M}\right)$, this latter used as internal standard (IS), as well as acetonitrile (LC-MS grade), ultra-pure water (LC-MS grade), and formic acid (MS grade 98\%) were provided by Sigma-Aldrich (Saint Louis, MO, USA). iPhree plates were purchased from Phenomenex (Torrance, CA, USA).

\subsection{Instrumentation}

Instrumental layout consisted of an AB Sciex API 4000 triple quadrupole mass spectrometer (Concord, ON, Canada), equipped with an electrospray (ESI) Turbo-V ion source, a quaternary HPLC pump (Series 200, PerkinElmer, Boston, MA, USA), and an Agilent 1290 Infinity UHPLC system (Santa Clara, CA, USA), consisting of a thermostated autosampler, a binary pump, and a column oven. A ten-port divert valve (Valco Instruments Co.Inc., Huston, TX, USA) allowed the diversion of eluent flow to waste, when necessary. Chromatographic separation was carried out by a Showa Denko K.K. (Tokyo, Japan) Shodex HILICpak VG-50 4E (5 $\mu \mathrm{m}, 250 \times 4.6$ mm ID) HPLC column, protected by a HILICpak VG-50 G 4A ( $5 \mu \mathrm{m}, 10 \times 4.6 \mathrm{~mm}$ ID) security guard cartridge. The AB Sciex Analyst version 1.6.3 software was used for instrument control and data acquisition and the AB Sciex Multiquant version 3.0.2 software for data processing. 


\subsection{Plasma samples}

Healthy volunteers (age $=33 \pm 9$ years; body mass index $(\mathrm{BMI})=23.1 \pm 2.9 \mathrm{~kg} / \mathrm{m}^{2}$; fasting plasma glucose $=5.2 \pm 0.4 \mathrm{mmol} / \mathrm{L})$ and patients with type 2 diabetes $(\mathrm{T} 2 \mathrm{D}$, age $=54 \pm 4$ years; $\mathrm{BMI}=27.8 \pm 2.3 ;$ diabetes duration $=14 \pm 6$ years; fasting plasma glucose $=12.0 \pm 1.7 \mathrm{mmol} / \mathrm{L} ;$ all mean \pm SD) were recruited at the Department of Clinical and Experimental Medicine, University of Pisa, Italy. After an overnight fast (10-12 hours), a blood sample was collected in EDTA tubes and centrifuged at 3,000 rpm for plasma separation.

\subsection{Sample storage and preparation}

Plasma samples were stored at $-20^{\circ} \mathrm{C}$. Before processing, each of them was thawed at room temperature, vortexed (15 min), and a $100 \mu \mathrm{L}$ aliquot was loaded into an iPhree 96 -well plate, where it was added with $400 \mu \mathrm{L}$ of a freshly prepared daily precipitation solution (DPS) containing acetonitrile, formic acid $0.1 \%(\mathrm{~V} \%)$, and IS at $3.125 \mu \mathrm{g} / \mathrm{mL}$ concentration level. The obtained suspension was eluted by a 96-well plate vacuum manifold and $5 \mu \mathrm{L}$ of the resulted eluate was injected into the LC-MS-MS system for the analysis.

\subsection{Preparation of calibrators and quality controls}

Solutions of mannose $(100 \mu \mathrm{g} / \mathrm{mL})$ and ${ }^{13} \mathrm{C}_{6}$-mannose $(100 \mu \mathrm{g} / \mathrm{mL})$ were stored at $-20{ }^{\circ} \mathrm{C}$ and used as standard stock solutions. The calibration curve was built using 8 standard solutions, prepared by serial dilution with water at the following concentration levels: $0.31\left(\mathrm{~L}_{1}\right), 0.63\left(\mathrm{~L}_{2}\right)$, $1.25\left(\mathrm{~L}_{3}\right), 2.50\left(\mathrm{~L}_{4}\right), 5.0\left(\mathrm{~L}_{5}\right), 10.0\left(\mathrm{~L}_{6}\right), 20.0\left(\mathrm{~L}_{7}\right)$ and $40.0 \mu \mathrm{g} / \mathrm{mL}\left(\mathrm{L}_{8}\right)$. Three quality control solutions, prepared by a proper water dilution of the mannose stock solution in order to get 2.0 $\left(\mathrm{QC}_{1}\right), 8.0\left(\mathrm{QC}_{2}\right)$, and $32.0 \mu \mathrm{g} / \mathrm{mL}\left(\mathrm{QC}_{3}\right)$ concentration levels, were analyzed in each batch. Both

calibrators and QCs (100 $\mu \mathrm{L}$ each) were added with DPS and submitted to the same treatment as the samples. 


\subsection{HPLC conditions}

Chromatographic separation was carried out under isocratic conditions, by delivering a solvent mixture consisting of acetonitrile ( $85 \%$, by volume) and water $(15 \%)$ by the binary pump, at a flowrate of $1000 \mu \mathrm{L} / \mathrm{min}$. A contribution to the robustness of the analytical method was provided by supplying eluate to the mass spectrometer just in the time range 9-12.6 min (which includes retention time of D-mannose), since both head and tail of the chromatographic run were diverted to waste by the ten-ports switching valve. On the contrary, in the time ranges 0-9 min and 12.6-16 min the quaternary pump supplied the ion source with a solvent mixture consisting of methanol (50\%, by volume) and water (50\%), at a flowrate of $100 \mu \mathrm{L} / \mathrm{min}$. A continuous solvent supply prevented the occurrence of unwanted electric discharges inside the source, avoiding damages of the mass spectrometer. The HPLC column was kept at a constant temperature of $40^{\circ} \mathrm{C}$ and injection volume for all samples, QCs, and calibrators was set to $5 \mu \mathrm{L}$.

\subsection{Mass spectrometry conditions}

The MS method was based on negative ion mode selected reaction monitoring (SRM) and made use of optimized collision energies (CEs) and collision exit potentials (CXPs). Two transitions for each compound were monitored, the more intense of which was used as a quantifier (Q) and the other one as a qualifier (q). These parameters are reported in Table 1. Further operative parameters were the following: declustering potential (DPs), $40 \mathrm{~V}$; entrance potential (EP), -10.2 V; IQ1 lens potential, $-10 \mathrm{~V}$; ionspray voltage (IS), $-4.5 \mathrm{kV}$; gas source 1 (GS1) zero air, 60; gas source 2 (GS1) zero air, 40; source temperature (TEM), $450{ }^{\circ} \mathrm{C}$; collision gas (CAD) nitrogen, operative pressure with $\mathrm{CAD}$ gas on, $4.8 \mathrm{mPa}$.

\subsection{Method Validation}

The method was validated in compliance with EMA guidelines with regard to selectivity, linearity, lower limit of detection (LLOD) and lower limit of quantitation (LLOQ), matrix effect, 
recovery, accuracy, precision, and stability [14]. Selectivity was evaluated by separate injection of water solutions of D-mannose, D-glucose, and D-galactose, as well as solutions containing all of them together. Retention time of each compound was monitored, in order to exclude a co-elution of D-mannose with its epimers. Linearity was assessed by calculating the correlation coefficient of 10 linear calibration curves, built as usual by 8 standard solutions at different levels, within the concentration range $0.31-40.00 \mu \mathrm{g} / \mathrm{mL}$. Instrumental LLOD and LLOQ, intended as the smallest concentration of D-mannose that can be reliably detected and quantified in a sample, respectively, were evaluated by injecting water solutions of D-mannose at decreasing concentration levels up to signals 3 and 10 times the noise level for LLOD and LLOQ, respectively, measured by a specific software tool included in the Analyst software. Ionization suppression or enhancement, commonly referred to as matrix effect (ME), could potentially be induced by the co-elution of D-mannose with the matrix components. A quantitative estimate of such an effect was performed according to the Matuszewski's assay method, by using ${ }^{13} \mathrm{C}_{6}$-mannose as a surrogate analyte [15]. In practice, the chromatographic peak area of ${ }^{13} \mathrm{C}_{6}$-mannose from a water solution (A) was compared to those from 9 different human plasma matrices (B), all of them treated in the same way as the real samples, except for ${ }^{13} \mathrm{C}_{6}$-mannose which was spiked after the iPhree elution, so that its final (i.e., injected) concentration was the same as the real samples $(2.5 \mu \mathrm{g} / \mathrm{mL})$. The matrix effect was then calculated for each human plasma as the ratio of the peak areas of ${ }^{13} \mathrm{C}_{6}$-mannose in matrix $\mathrm{B}$ and in solution $\mathrm{A}$, by the formula $\mathrm{ME}(\%)=\mathrm{B} / \mathrm{A} \times 100$. Recovery $(\mathrm{RE})$ was evaluated by processing data from 3 different experiments: a) comparison between water solutions of ${ }^{13} \mathrm{C}_{6}$-mannose, prepared by spiking proper amounts of ${ }^{13} \mathrm{C}_{6}$-mannose before the iPhree treatment $\left(\mathrm{C}_{1}\right)$ or after the elution $\left(\mathrm{D}_{1}\right)$, in order to obtain solutions with $2.5 \mu \mathrm{g} / \mathrm{mL}$ as a final concentration; b) comparison between real human plasma from healthy volunteers added with ${ }^{13} \mathrm{C}_{6}$-mannose before the iPhree treatment $\left(\mathrm{C}_{2}\right)$ or after, i.e., to the iPhree eluate $\left(\mathrm{D}_{2}\right)$, such as its final concentration was $2.5 \mu \mathrm{g} / \mathrm{mL}$; c) comparison between water solutions prepared by spiking mannose before $\left(\mathrm{C}_{3}\right)$ and after $\left(\mathrm{D}_{3}\right)$ the iPhree treatment, in order to get mannose at the same final concentrations of QCs, i.e., $0.4\left(\mathrm{QC}_{1}\right), 1.6$ 
$\left(\mathrm{QC}_{2}\right)$, and $6.4 \mu \mathrm{g} / \mathrm{mL}\left(\mathrm{QC}_{3}\right)$. In each experiment recovery was calculated as the ratio of the peak areas of ${ }^{13} \mathrm{C}_{6}$-mannose or mannose in matrix $\mathrm{C}$ and matrix $\mathrm{D}$ by the formula $\mathrm{RE}(\%)=\mathrm{C} / \mathrm{D} \times 100$. Moreover, the evaluation of recovery in experiment c) was carried out also by normalizing mannose peak area with the peak area of ${ }^{13} \mathrm{C}_{6}$-mannose, which was added to the QCs as an IS, according to the procedure described in the "Preparation of calibrators and quality controls" section. Thus, recovery was calculated by the formula $\left.\mathrm{RE}(\%)=\left[\left(\mathrm{C} / \mathrm{C}_{\mathrm{IS}}\right) /\left(\mathrm{D} / \mathrm{D}_{\mathrm{IS}}\right)\right] \times 100\right)$. Intra-day accuracy (expressed as accuracy \%) and precision (expressed as relative standard deviation \%, RSD \%) were measured on a human plasma sample from a healthy subject, spiked with mannose prior to the usual sample preparation in order to achieve additions at 3 different concentration levels $\left(\mathrm{C}_{1}, 6.25 \mu \mathrm{g} / \mathrm{mL}\right.$; $\left.\mathrm{C}_{2}, 12.50 \mu \mathrm{g} / \mathrm{mL} ; \mathrm{C}_{3}, 25.00 \mu \mathrm{g} / \mathrm{mL}\right)$. The added mannose was estimated by two different formulas: d) $\mathrm{C}_{n}=\mathrm{C}_{\mathrm{Tn}}-\mathrm{C}_{\mathrm{B}}$, i.e., the measured endogenous concentration in the blank plasma sample $\left(\mathrm{C}_{\mathrm{B}}\right)$ was subtracted from the total concentration $\left(\mathrm{C}_{\mathrm{Tn}}\right.$, endogenous + added concentration) in each spiked sample to determine the concentration value of added mannose $\left(C_{n}\right.$ indicates $C_{1}, C_{2}$, or $\left.\left.C_{3}\right) ; e\right) C_{n}=$

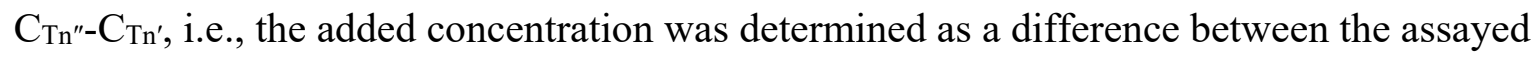
concentration values of 2 differently spiked plasma aliquots. All the concentrations were assayed by 10 replicate injections, and the statistical parameters were calculated by using mean concentration values for the subtrahends in the above reported formulas. Moreover, intra-day accuracy and precision were also assessed using 6 replicate injections of low, medium and high concentration QCs; intra-day accuracy and precision were measured on 3 different days and were considered acceptable when within $\pm 15 \%$. Stability of D-mannose as a result of a freeze-thaw cycle was evaluated. An aliquot of freshly prepared low, medium, and high QCs, as well as several plasma samples, were immediately injected into the HPLC-MS-MS system, and the results were compared to those from a second aliquot of the same QCs and samples frozen at $-20{ }^{\circ} \mathrm{C}$ (stored for 30 days) and thawed at room temperature before sample preparation and assay.

\section{Results}




\subsection{Method Validation}

HPLC provided an adequate separation of mannose from its main interferents glucose and galactose (Figure 1), ensuring the necessary selectivity to the method. The calibration curve, which was built by plotting the peak area ratio (y) of the analyte to IS versus the nominal concentration (x) of the analyte, with a weighed $(1 / x)$ linear regression, was linear within the acquired concentration range $(0.31-40.0 \mu \mathrm{g} / \mathrm{mL})$, with a correlation coefficient always greater than 0.997 . The values $($ mean $\pm \mathrm{SD} ; \mathrm{n}=10)$ of its slope and intercept were $\mathrm{m}=0.091 \pm 0.029$ and $\mathrm{q}=0.0206 \pm 0.0716$, respectively. Instrumental LLOD and LLOQ were 0.31 and $1.25 \mu \mathrm{g} / \mathrm{mL}$, respectively, i.e., LLOQ was at least 6 times lower than the lowest mannose concentration in our samples. The matrix effect, tested on nine different human plasma matrices and expressed as mean $\pm \mathrm{SD} \%$, was found to be $62.2 \pm 5.4 \%$. As a consequence, signal was clearly detectable in all the matrices, although it was suppressed by about $38 \%$. Recovery, tested with ${ }^{13} \mathrm{C}_{6}$-D-mannose at $2.5 \mu \mathrm{g} / \mathrm{mL}$ as a final concentration and expressed as mean $\pm \mathrm{SD} \%$, was $91.5 \pm 4.7 \%$ in water solutions (experiment a) and $62.5 \pm 4.5 \%$ in human plasma (experiment $b$ ). The addition of mannose to aqueous solutions before and after the iPhree treatment (experiment $c$ ) in order to get it at a final concentrations of 0.4 $\left(\mathrm{QC}_{1}\right), 1.6\left(\mathrm{QC}_{2}\right)$, and $6.4 \mu \mathrm{g} / \mathrm{mL}\left(\mathrm{QC}_{3}\right)$ provided mean recoveries of $83.4 \%, 87.4 \%$, and $95.5 \%$, respectively, which increased up to $89.1 \%, 100 \%$, and $98.8 \%$ when mannose peak areas were normalized by the IS peak areas. Intra-day and inter-day accuracy assessed on the three quality controls in water solution, was in the range $96.07-103.68 \%$, while RSD was $<9.81$. The abovementioned intra-day accuracy was confirmed by further experiments on a real human plasma sample spiked with mannose at $6.25 \mu \mathrm{g} / \mathrm{mL}\left(\mathrm{C}_{1}\right), 12.50 \mu \mathrm{g} / \mathrm{mL}\left(\mathrm{C}_{2}\right)$, and $25.00 \mu \mathrm{g} / \mathrm{mL}\left(\mathrm{C}_{3}\right)$ concentration levels, which gave 96.59 - $101.75 \%$ as an accuracy range. Also precision was essentially confirmed, as RSD \% always resulted $<4.59$. Accuracy and precision data are all reported in Table 2. Stability of D-mannose under one freeze $\left(-20^{\circ} \mathrm{C}, 30\right.$ days)-thaw (room temperature) cycle for low, medium and high QC was $95.19 \pm 4.11 \%$ (variation \pm SD), while it was $92.40 \pm 1.66 \%$ when detected on 5 different plasma samples. 


\subsection{Concentration values}

Twenty healthy subjects and twenty diabetic patients were assayed in order to establish the reference intervals. The endogenous D-Mannose concentration, expressed as mean $\pm \mathrm{SD}$, in healthy volunteers was $9.93 \pm 3.37 \mu \mathrm{g} / \mathrm{mL}$, while in patients with type 2 diabetes it averaged $23.47 \pm 6.19$ $\mu \mathrm{g} / \mathrm{mL}(\mathrm{p}<0.0001$ by Mann-Whitney $U$ test $)$.

\section{Discussion}

\subsection{Method Performances}

The HPLC-MS-MS method was based on the isotope dilution technique [16] and made use of a stable isotope labeled analogue of D-mannose as an IS, i.e., ${ }^{13} \mathrm{C}_{6}$-D-mannose. This compound has equivalent physical-chemical properties of mannose and, therefore, the same instrumental response. As an IS, it compensates for the variability of some critical parameters, such as MS ionization efficiency, sample stability, and extraction recovery during samples preparation [17]. Chromatography of D-mannose in biological samples is complicated by the challenging separation from its endogenous epimers D-glucose and D-galactose, which often requires ad hoc strategies and specific materials [10]. In a recently published HPLC-MS-MS method, White et al. [4] proposed the use of a Supelcogel Pb (Sigma-Aldrich, Saint Louis, MO, USA) column capable of separating sugars epimers by means of an ion exchange resin, which interacts with the analytes by both size exclusion and ligand exchange, the latter mechanism probably prevailing in the case of D-mannose. In the course of the present study, a Rezex RCM Monosaccharide $\mathrm{Ca}^{++}$(Phenomenex, Torrance, CA, USA) $300 \times 7.8 \mathrm{~mm}$ column [18], containing an ion exclusion material similar to that of Supelcogel $\mathrm{Pb}$, was extensively tested by running more than 100 samples. It provided the needed chromatographic separation, but its performance degraded quite rapidly for the progressive deactivation of the stationary phase, so that a frequent column regeneration was necessary in order to prevent a significant decrease in the separation capability. The analytical method centered on 
this column showed further limitations, such as long run-times, which made it less than ideal for its routine use, and the use of just water as a mobile phase, which was unable to remove most of the organic residues adsorbed on the packing material. Also the Shodex HILICpak VG-50 4E, a polymer-based amino column for hydrophilic interaction chromatography (HILIC) that was next tested and then adopted for our definitive method, provided an adequate separation of mannose from galactose and glucose (Figure 1). A further structural isomer of mannose, sometimes regarded as a potential interferent, is fructose. It has a significantly different structure and was easily separated from mannose by the HILICpak column. Interestingly, also the HILICpak column exhibited a progressive degradation of the separation efficiency, but it evolved much slower with respect to the Phenomenex Rezex column and could be overcome by an occasional column washing in alkaline solution $(0.1 \mathrm{M} \mathrm{NaOH})$. In addition, the HILICpak column had a longer lifetime.

Instrumental sensitivity was good enough for our aims, with $1.25 \mu \mathrm{g} / \mathrm{mL}$ as a LLOQ, which was at least 6 times lower than the lowest mannose concentration in our samples. This value is not impressive, although in a good agreement with data from the literature, as White et al. [4] gave 1.0 $\mu \mathrm{g} / \mathrm{mL}$ as a LLOQ. However, this result was affected by a 5 -fold sample dilution, that was included in sample preparation step in order to increase the method ruggedness, and by the broadness of the HPLC peak ( $40 \mathrm{sec}$.), which anyway is a quite common behavior with monosaccharides $[4,11]$.

Matrix effect was non-negligible, as signal suppression was about 38\% [19], but it was not critical and there was no need to improve the sample clean-up procedure. This value disagrees with the results from White et al. [4], who got an insignificant signal suppression by using a sample preparation based just on protein precipitation. Such an outcome might be surprising if matrix effect had been assessed on real plasma or serum samples rather than on a surrogate sample obtained by spiking D-mannose to a blank matrix made by a 4\% BSA in PBS, as White et al. did.

Recovery, assessed by ${ }^{13} \mathrm{C}_{6}$-D-mannose at $2.5 \mu \mathrm{g} / \mathrm{mL}$ as a final concentration, was significantly higher in water solutions than in human plasma (91.5 $\pm 4.7 \%$ vs. $62.5 \pm 4.5 \%)$, suggesting that 
protein precipitate from plasma could hold some ${ }^{13} \mathrm{C}_{6}$-D-mannose, thereby preventing elution of its total amount through the stationary phase. In addition, recovery was influenced by the analyte concentration, since mannose in $\mathrm{QC}_{1}$ (injected concentration, $0.4 \mu \mathrm{g} / \mathrm{mL}$ in water), $\mathrm{QC}_{2}(1.6$ $\mu \mathrm{g} / \mathrm{mL})$, and $\mathrm{QC}_{3}(6.4 \mu \mathrm{g} / \mathrm{mL})$ exhibited mean recoveries of $83.4 \%, 87.4 \%$, and $95.5 \%$, which improved up to $89.1 \%, 100 \%$, and $98.8 \%$, respectively, when the mannose peak areas were normalized by the IS peak areas. Also recovery in human plasma was a little disadvantageous with respect to other method in the literature $[4,10,11]$, but since LLOQ was more than acceptable, no implementation was made to the sample preparation procedure in order to preserve its simplicity and the method suitability for a routine use.

Intra-day accuracy and precision were assessed by experiments on either human plasma added with known amounts of mannose or water solutions with mannose at 3 different concentration levels, namely QCs. Since no mannose-free plasma was available to be used as a blank matrix, a sample containing endogenous mannose at a relatively low concentration level, i.e., $7.37 \mu \mathrm{g} / \mathrm{mL}$, was used in its place and was spiked with mannose at 3 final concentration levels, i.e., $6.25 \mu \mathrm{g} / \mathrm{mL}$ $\left(\mathrm{C}_{1}\right), 12.50 \mu \mathrm{g} / \mathrm{mL}\left(\mathrm{C}_{2}\right)$, and $25.00 \mu \mathrm{g} / \mathrm{mL}\left(\mathrm{C}_{3}\right)$. The added concentrations were then estimated by subtracting the mean assayed endogenous concentration from the total assayed concentrations $\left(\mathrm{C}_{\mathrm{n}}=\right.$ $\mathrm{C}_{\mathrm{Tn}}-\mathrm{C}_{\mathrm{B}}$ ) and by calculating the difference between the total assayed concentrations of two sample aliquots spiked with different amounts of mannose $\left(\mathrm{C}_{n}=\mathrm{C}_{\mathrm{Tn}^{\prime \prime}-}-\mathrm{C}_{\mathrm{Tn}^{\prime}}\right)$. As reported in Table 2, all results, obtained by calibration curves built with calibrators in water solution, are within the acceptance range $( \pm 15 \%)$. Positive outcomes came also from QCs, which were also used to assess inter-day accuracy and precision, with similar results (Table 2). These results were satisfactory and in a substantial agreement with data in the literature [4] and in accordance to the EMA guidelines [14]. 
When it is contained in human plasma, D-mannose under one freeze-thaw cycle is not completely stable and undergoes to a decrease in its concentration of $7.6 \%$ on average. Hence, freshly prepared sample should be processed whenever possible.

\subsection{Concentration values}

Most of the mannose concentration ranges in the literature refers to assay methods not based on HPLC-MS-MS, sometimes to serum specimens or to subjects of different ethnicity. Hence, the comparison between data in the literature and our reference plasma concentration could be merely indicative. Actually, in plasma specimens from healthy volunteers Pitkänen measured a concentration of $7.0 \pm 2.2 \mu \mathrm{g} / \mathrm{mL}$ by gas-chromatography coupled to mass spectrometry (GC-MS) [12], Józwik et al. a concentration of $10.0 \pm 0.4 \mu \mathrm{g} / \mathrm{mL}$ in samples from women in a preovulatory period by HPLC coupled to an amperometric detector [20], Taguchi et al. and Yoshimura et al. levels of $6.4 \pm 2.3 \mu \mathrm{g} / \mathrm{mL}$ and $7.4 \pm 0.4 \mu \mathrm{g} / \mathrm{mL}$, respectively, by using HPLC with a fluorescence detector [10,21], Sone et al. a concentration of $7.4 \pm 1.6 \mu \mathrm{g} / \mathrm{mL}$ by a colorimetric based technique [22], and Akazawa et al. 9. $8 \pm 0.4 \mu \mathrm{g} / \mathrm{mL}$ by enzymatic assay on samples collected during weeks 34-40 of human pregnancy [23]. Serum from healthy volunteers usually provided lower values, although of the same order of magnitude. In particular, White et al., who also used an HPLC-MSMS based method, assayed a concentration of $6.3 \pm 3.6 \mu \mathrm{g} / \mathrm{mL}$ [4], Charcon et al. a level of $5.4 \pm$ $2.3 \mu \mathrm{g} / \mathrm{mL}$ on paediatric samples using capillary electrophoresis with a fluorescence detector [13], Soyama et al. and Etchison et al. values of $3.8 \pm 2.9 \mu \mathrm{g} / \mathrm{mL}$ and $5.4 \pm 1.8 \mu \mathrm{g} / \mathrm{mL}$, respectively, by enzymatic assay $[7,8]$. Data in the literature show higher concentrations levels of plasma mannose in samples from patients with type 2 diabetes. Actually, Pitkänen detected mannose at a concentration level of $21.3 \pm 10.5 \mu \mathrm{g} / \mathrm{mL}$ [12], Yoshimura et al. at $8.1 \pm 2.3 \mu \mathrm{g} / \mathrm{mL}$ [21], Sone et al. at $9.3 \pm 2.4 \mu \mathrm{g} / \mathrm{mL}$ [22], and Akazawa et al. at $16.9 \pm 0.6 \mu \mathrm{g} / \mathrm{mL}$ [23]. It's worth noting that our results are consistent with the plasma concentration ranges proposed by Pitkänen, which were also obtained by mass spectrometry, although coupled to gas-chromatography, i.e., $9.93 \pm 3.37 \mu \mathrm{g} / \mathrm{mL}$ 
vs. $7.0 \pm 2.2 \mu \mathrm{g} / \mathrm{mL}$ in healthy volunteers, and $23.47 \pm 6.19 \mu \mathrm{g} / \mathrm{mL}$ vs. $21.3 \pm 10.5 \mu \mathrm{g} / \mathrm{mL}$ in patients with type 2 diabetes.

\section{Conclusions}

We developed a simple and reliable LC-MS-MS method for the quantification of D-mannose in human plasma, which was validated in compliance with the EMA guidelines [14] to be used in clinical laboratories for accurate assays. The method provided satisfactory results in terms of selectivity, as it distinguished D-mannose from its epimers glucose and galactose, reproducibility, with RSD always $<10 \%$, and accuracy, in the range $96-104 \%$. Linearity was good in the concentration range of interest, i.e., $0.31-40 \mu \mathrm{g} / \mathrm{mL}$, and instrumental LLOD and LLOQ were 0.31 and $1.25 \mu \mathrm{g} / \mathrm{mL}$, respectively. The latter value was at least 6 times lower of the lowest concentration assayed in human plasma samples, confirming that sensitivity was adequate for the purpose, although not impressive. It often happens with the separation of sugar epimers, as chromatography often yields rather broad peaks which affect the method sensitivity. Moreover, the sensitivity of our method suffered from the 5-fold sample dilution during sample preparation, which anyway improved the method ruggedness. Another point of interest was the use of calibrators in water solution, which simplified the pre-analytical procedure.

The method was used for the assessment of D-mannose in plasma samples obtained from diabetic and non-diabetic subjects. Our preliminary data on fasting samples document the presence of higher plasma mannose concentrations in type 2 diabetes, par excellence an insulin resistant state, thereby confirming the findings obtained by screening metabolomics [6]. Extensive clinical investigation into the mechanisms of this increase proves that physiological hyperinsulinemia is a potent negative regulator of circulating mannose levels, and that this insulin effect is blunted in patients with type 2 diabetes [24]. Therefore, D-mannose could be further tested a new robust biomarker of insulin resistance and insulin sensitivity in large-scale studies. 


\section{Author Contributions Statement}

$\mathrm{BC}$ designed and performed the experiments, and carried out data analysis; SC, NB performed the experiments; SB collected the human samples; RZ critically revised the manuscript; EF supervised the experimental work and contributed funding; AS designed and supervised the experimental work, carried out data interpretation, and wrote the manuscript draft. All authors approved the final submitted version of the manuscript.

\section{Conflict of Interest Statement}

The work was not carried out in the presence of any personal, professional or financial relationships that could potentially be construed as a conflict of interest.

\section{Ethics statements}

Plasma was obtained by using the remaining part of samples drawn for independent clinical indications. All subjects gave informed consent and since no additional blood drawings were performed, ethical committee approval was not required.

\section{Funding}

This study was funded by the Innovative Medicines Initiative Joint Undertaking under EMIF grant agreement no. 115372 . 


\section{References}

[1] H.H. Freeze, V. Sharma, Metabolic manipulation of glycosylation disorders in humans and animal models, Semin. Cell Dev. Biol. 21 (2010) 655-662.

https://doi.org/10.1016/j.semcdb.2010.03.011.

[2] P.T. Martin, Congenital muscular dystrophies involving the O-mannose pathway, Curr. Mol. Med. 7 (2007) 417-425. https://doi.org/10.2174/156652407780831601.

[3] B. Sanchez-Espiridion, D. Liang, J.A. Ajani, S. Liang, Y. Ye, M.T.A. Hildebrandt, J. Gu, X. $\mathrm{Wu}$, Identification of serum markers of esophageal adenocarcinoma by global and targeted metabolic profiling, Clin. Gastroenterol. Hepatol. 13 (2015) 1730-1737. https://doi.org/10.1016/j.cgh.2015.05.023.

[4] L. White, J. Ma, S. Liang, B. Sanchez-Espiridion, D. Liang, LC-MS/MS determination of Dmannose in human serum as a potential cancer biomarker, J. Pharm. Biomed. Anal. 137 (2017) 54-59. https://doi.org/10.1016/j.jpba.2016.12.017.

[5] E. Jobard, C. Pontoizeau, B.J. Blaise, T. Bachelot, B. Elena-Herrmann, O. Tredan, A serum nuclear magnetic resonance-based metabolomic signature of advanced metastatic human breast cancer, Cancer. Lett. 343 (2014) 33-41. https://doi.org/10.1016/j.canlet.2013.09.011.

[6] A. Mardinoglu, A. Stančáková, L.A. Lotta, J. Kuusisto, J. Boren, M. Blüher, N.J. Wareham, E. Ferrannini, P.H. Groop, M. Laakso, C. Langenberg, U. Smith, Plasma mannose levels are associated with incident type 2 diabetes and cardiovascular disease, Cell metabolism. 26 (2017) 281-283. https://doi.org/10.1016/j.cmet.2017.07.006.

[7] K. Soyama, Enzymatic determination of D-mannose in serum, Clin. Chem. 30 (1984) 293294.

[8] J.R. Etchison, H.H. Freeze, Enzymatic assay of D-mannose in serum, Clin. Chem. 43 (1997) $533-538$. 
[9] E. Pitkänen, O. Pitkänen, L. Uotila, Enzymatic determination of unbound D-mannose in serum, Eur. J. Clin. Chem. Clin. Biochem. 35 (1997) 761-766. https://www.ncbi.nlm.nih.gov/pubmed/9368794.

[10] T. Taguchi, I. Miwa, T. Mizutani, H. Nakajima, Y. Fukumura, I. Kobayashi, M. Yabuuchi, I. Miwa, Determination of D-mannose in plasma by HPLC, Clin. Chem. 49 (2003) 181-183. https://doi.org/10.1373/49.1.181.

[11] I. Miwa, T. Taguchi, A simple HPLC assay for plasma D-mannose, Clin. Chim. Acta 422 (2013) 42- 43. https://doi.org/10.1016/j.cca.2013.04.005.

[12] E. Pitkänen, Mannose mannitol, fructose and 1,5-anhydroglucitol concentrations measured by gas chromatography/mass spectrometry in blood plasma of diabetic patients, Clin. Chim. Acta 251 (1996) 91-103.

[13] H.A. Carchon, J. Jaeken, Determination of D-mannose in serum by capillary electrophoresis, Clin. Chem. 47 (2001) 1319-1321.

[14] European Medicines Agency, Guideline on bioanalytical method validation. http://www.ema.europa.eu/docs/en_GB/document_library/Scientific_guideline/2011/08/WC5 00109686.pdf, 2015 (adopted 21 July 2011).

[15] B.K. Matuszewski, M.L. Constanzer, C.M. Chavez-Eng, Strategies for the Assessment of Matrix Effect in Quantitative Bioanalytical Methods Based on HPLC-MS/MS, Anal. Chem. 75 (2003) 3019-3030. https://doi.org/10.1021/ac020361s.

[16] E. Ciccimaro, I.A. Blair, Stable-isotope dilution LC-MS for quantitative biomarker analysis, Bioanalysis 2 (2010) 311-341. https://doi.org/10.4155/bio.09.185.

[17] J. Wieling, LC-MS-MS experiences with internal standards, Chromatographia 55 (2002) 107-113. https://doi.org /10.1007/BF02493365.

[18] X. Wu, S. Staggenborg, J.L. Propheter, W.L. Rooney, J. Yu, D. Wang, Features of sweet sorghum juice and their performance in ethanol fermentation, Ind. Crops Prod. 31 (2010) 164170. https://doi.org/10.1016/j.indcrop.2009.10.006. 
[19] A. Furey, M. Moriarty, V. Bane, B. Kinsella, M. Lehane, Ion suppression; a critical review on causes, evaluation, prevention and applications, Talanta 115 (2013) 104-122. https://doi.org/10.1016/j.talanta.2013.03.048.

[20] M. Józwik, M. Józwik, C. Teng, F.C Battaglia, Concentrations of monosaccharides and their amino and alcohol derivatives in human preovulatory follicular fluid, Mol. Hum. Reprod. 13 (2007) 791-796. https://doi.org/10.1093/molehr/gam060.

[21] K. Yoshimura, S. Hirano, H. Takata, S. Funakoshi, S. Ohmi, E. Amano, Y. Nishi, M. Inoue, Y. Fukuda, H. Hayashi, T. Taguchi, S. Yamada, I. Miwa, Y. Terada, S. Fujimoto, Plasma mannose level, a putative indicator of glycogenolysis, and glucose tolerance in Japanese individuals, J. Diabetes Investig. 8 (2017) 489-495. https://doi.org/10.1111/jdi.12622.

[22] H. Sone, H. Shimano, H. Ebinuma, A. Takahashi, Y. Yano, K.T. Iida, H. Suzuki, H. Toyoshima, Y. Kawakami, Y. Okuda, Y. Noguchi, K. Ushizawa, K. Saito, N. Yamada, Physiological changes in circulating mannose levels in normal, glucose-intolerant, and diabetic subjects, Metab. Clin. Exp. 52 (2003) 1019-1027. https://doi.org/10.1016/S00260495(03)00153-7.

[23] S. Akazawa, B.E. Metzger, N. Freinkel, Relationships between glucose and mannose during late gestation in normal pregnancy and pregnancy complicated by diabetes mellitus: concurrent concentrations in maternal plasma and amniotic fluid, J. Clin. Endocrinol. Metab. 62 (1986) 984-989. https://doi.org/10.1210/jcem-62-5-984.

[24] E. Ferrannini, M. Bokarewa, S. Hedjazifar, K. Andersson, S. Baldi, B. Campi, E. Muscelli, A. Saba, I. Sterner, C. Wasen, U. Smith, Mannose is an insulin-regulated metabolite reflecting whole-body insulin sensitivity in man. Submitted. 
Table 1. Mass spectrometry operative parameters

\begin{tabular}{ccccc}
\hline \multirow{2}{*}{ Analyte } & \multicolumn{4}{c}{ Operative Parameters } \\
\cline { 2 - 5 } & SRM transition & CE & CXP \\
\hline \multirow{2}{*}{ Mannose } & q & $178.9 \rightarrow 59.0$ & -24 & -4.0 \\
& Q & $178.9 \rightarrow 88.9$ & -11 & -6.0 \\
Mannose- ${ }^{13} \mathrm{C}_{6}$ & q & $184.9 \rightarrow 60.9$ & -28 & -9.3 \\
& Q & $184.9 \rightarrow 92.0$ & -12 & -15.0 \\
\hline
\end{tabular}


Table 2. Accuracy and precision of D-mannose assay.

\begin{tabular}{ccrrrr}
\hline \multirow{6}{*}{ Intra-day } & & $\begin{array}{c}\text { Nominal Conc. } \\
(\mu g / m l)\end{array}$ & $\begin{array}{c}\text { Mean Conc. } \\
(\mu g / m l)\end{array}$ & RSD (\%) & Accuracy (\%) \\
\cline { 3 - 6 } & $\mathrm{C}_{1}-\mathrm{C}_{\mathrm{B}}$ & 6.25 & 6.19 & 3.69 & 98.97 \\
& $\mathrm{C}_{2}-\mathrm{C}_{\mathrm{B}}$ & 12.50 & 12.22 & 2.27 & 97.78 \\
& $\mathrm{C}_{3}-\mathrm{C}_{\mathrm{B}}$ & 25.00 & 24.94 & 0.95 & 99.77 \\
& $\mathrm{C}_{2}-\mathrm{C}_{1}$ & 6.25 & 6.04 & 4.59 & 96.59 \\
& $\mathrm{C}_{3}-\mathrm{C}_{2}$ & 12.50 & 12.72 & 1.86 & 101.75 \\
& $\mathrm{C}_{3}-\mathrm{C}_{1}$ & 18.75 & 18.76 & 1.26 & 100.03 \\
& $\mathrm{QC}_{1}$ & 2.00 & 1.92 & 6.27 & 96.07 \\
& $\mathrm{QC}_{2}$ & 8.00 & 8.23 & 2.43 & 102.98 \\
& $\mathrm{QC}_{3}$ & 32.00 & 32.38 & 2.09 & 101.21 \\
\cline { 2 - 5 } Inter-day & 2.00 & 2.01 & 9.81 & 100.25 \\
& $\mathrm{QC}_{1}$ & 8.29 & 2.69 & 103.68 \\
& $\mathrm{QC}_{2}$ & 32.40 & 1.48 & 101.25 \\
\hline
\end{tabular}




\section{Figure legend}

Figure 1 - SRM chromatograms of m/z $178.9 \rightarrow 88.9$ Da from standard solutions containing mannose (a), galactose (b), and glucose (c) at a concentration of $1 \mu \mathrm{g} / \mathrm{mL}$. 